\title{
EEG Processing to Discriminate Transitive-Intransitive Motor Imagery Tasks: Preliminary Evidences using Support Vector Machines
}

\author{
Vincenzo Catrambone*, Alberto Greco, Giuseppe Averta, Matteo Bianchi, Nicola Vanello, \\ Antonio Bicchi, Gaetano Valenza, and Enzo Pasquale Scilingo
}

\begin{abstract}
It is known that brain dynamics significantly changes during motor imagery tasks of upper limb involving different kind of interactions with an object. Nevertheless, an automatic discrimination of transitive (i.e., actions involving an object) and intransitive (i.e., meaningful gestures that do not include the use of objects) imaginary actions using EEG dynamics has not been performed yet. In this study we exploit measures of EEG spectra to automatically discern between imaginary transitive and intransitive movements of the upper limb. To this end, nonlinear support vector machine algorithms are used to properly combine EEG-derived features, while a recursive feature elimination procedure highlights the most discriminant cortical regions and associated EEG frequency oscillations.
\end{abstract}

Results show the significance of $\gamma(30-45 \mathrm{~Hz})$ oscillations over the fronto-occipital and ipsilateral-parietal areas for the automatic classification of transitive-intransitive imaginary upper limb movements with a satisfactory accuracy of $70.97 \%$.

\section{INTRODUCTION}

Human body is indeed an extraordinary source of inspiration for the design of artificial systems [1], with special focus on the hand and arm as the primary tools humans rely on to interact with the environment, with important for applications in rehabilitation, simplified design and control guidelines, advanced human-robot interaction [2], [3].

The investigation of the underpinning neural mechanisms of human upper limb control plays a crucial role in the medical, neuroscience, and biomechanical research fields [1]. The sensory-motor analysis of human upper limb has received a lot of attention recently, and has been tackled from different perspective, such as kinematic [4], [5], biomechanical [6], [7], and neurological [8], [9]. More specifically, under a neurological point of view, upper limb movements can reasonably be seen as cognitive tasks and characterised according to neuro-physiological criteria. To this extent, several previous studies have investigated the topography and dynamics of brain activations during motor imagery, motor visualisation, and actual execution using different neuro-imaging techniques: e.g., functional magnetic resonance imaging [10]-[12], near infra-red spectroscopy [13], magneto-electroencephalography [14], [15] and electroencephalography (EEG) [16]-[19].

This research has received funding from the European Union's Horizon 2020 Research and Innovation Programme under Grant Agreement No.688857 (SoftPro).

Authors are with the Department of Information Engineering \& Bioengineering and Robotics Research Center E. Piaggio, School of Engineering, University of Pisa, Pisa, Italy.

* Corresponding author e-mail:

vincenzo. catramboneding.unipi.it
In one of these EEG-based studies, a crucial role of central cortex region has been highlighted during hand movement execution [16], especially regarding EEG oscillations in the $\alpha$ and $\beta$ bands. Other interesting findings have shown a significant relationship between frontal lobes and behavioural and communicative tasks executed through the upper limb [20]. Furthermore, other imaging studies have proven a correlation between movements and contralateral neural activity, and an uncorrelated remarkable activation of the ipsilateral hemisphere related to hand position representation [21]. On the other hand, the role of parietal and pre-motor cortex in both motion execution, imagery and observation has been well demonstrated [22]-[24].

In the task space, it is possible to identify three main classes of actions associated with different neuropsychological correlates [8], [25], [26]: intransitive (i.e., meaningful gestures that do not include the use of objects), transitive (i.e., actions involving an object), and toolmediated (i.e., actions in which an object is used to act upon another) [2]. A functional imaging study [27] has already demonstrated that these three classes of actions generate distinct neural patterns in the human brain during motor visualisation, and exhibit a specific topographical organisation of the brain response. However, to the best of our knowledge no study has been proposed with the aim of investigating possible differences in EEG dynamics capable to distinguish these classes of movement.

In light of the above, here we present a preliminary study aiming to automatically recognise two different classes of upper limb movements, i.e., transitive and intransitive, using only the information coming from frequency analysis of EEG. In this analysis, we focused on the time slot where the subjects were assumed to only imagine the movements, before the actual motor execution.

\section{Materials AND Methods}

\section{A. Experimental dataset}

Thirty-three young healthy volunteers (26.6 years on average, 17 females, all right handed) were recruited among students of the University of Pisa. All experimental procedures were approved by the local ethical committee. Each subject was asked to imagine and then actually perform 20 specific right upper limb movements three times, therefore performing a total of 60 motor tasks. These tasks were divided into two categories depending on the kind of interaction with objects: (i) transitive for tool-based tasks (e.g., reach and grasp a cup and mimic drinking); (ii) intransitive 


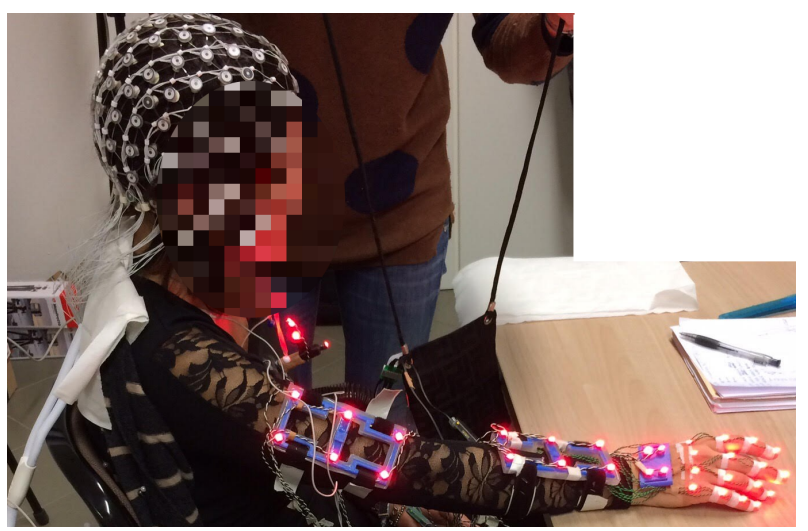

Fig. 1: Exemplary experimental set-up. The subject is equipped with high resolution EEG sensors and active optical markers for motion tracking.

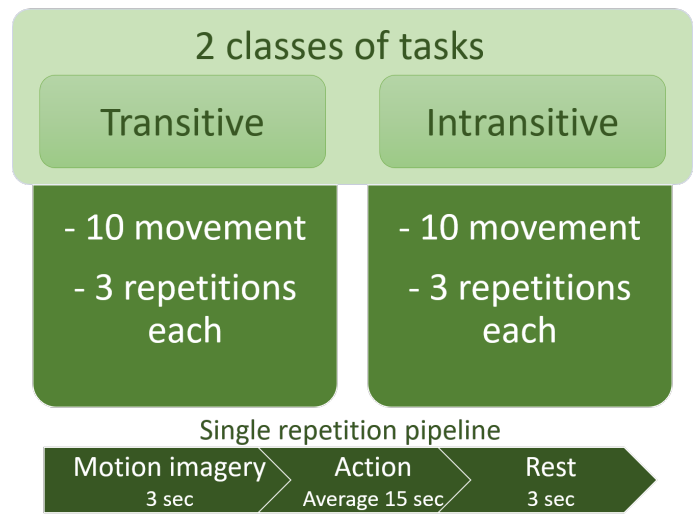

Fig. 2: Logic scheme of the experimental protocol timeline comprising 3 repetitions of 10 transitive, intransitive, and tool-mediated movements. Each task included a first motor imagery phase, an actual movement recording, and a final resting state

for object-free movements (e.g., thumb down). Throughout the experiment we performed both the optical registration of the upper limb movements and the EEG data acquisition. For the former we used ten stereo-cameras tracking the 3D position of markers attached to upper limb with a Sampling rate of $480 \mathrm{~Hz}$, whereas for the latter we used a 128 channels Geodesic EEG Systems 300 from the Electrical Geodesics Inc., with a sampling rate of $500 \mathrm{~Hz}$. For each task, the subject was first instructed on the movement to be performed, which was mimed by the operator. Afterwards, the subject was asked to imagine the movement for $3 \mathrm{~s}$ and then to actually perform it. A final $3 \mathrm{~s}$ resting state completed the task (see Figure 2).

\section{B. EEG analysis}

The EEG processing chain comprised filtering, segmentation, artefacts detection and removal, data re-referencing, and bad channels interpolation. Frequency filtering was designed using a band-pass finite impulse response filter between $0.5 \mathrm{~Hz}$ and $45 \mathrm{~Hz}$ with a Butterworth approximation. Signals were then segmented into time windows in correspondence with the start and end of each trial, and the first $3 \mathrm{~s}$ of motor imagery task were retained for further analyses. An independent component analysis decomposition (FastICA) was applied, and independent components related to eyeblinks, heart and muscles electrical activity, head and arm movements were discarded after visual inspection. Then, signals were re-referenced to the average of all channels for each time sample. Finally, corrupted channels, defined as signals with unexpected events and with high-frequency noise, were interpolated using spherical interpolation algorithm applied to the closest electrodes. The EEGLAB toolbox [28] for Matlab was used for most of the aforementioned processing operations.

\section{Spectral Analysis}

The power spectral density (PSD) was estimated from each EEG channel signal using the Welch's method, which averaged the power values across moving, overlapping $1 \mathrm{~s}$ time windows. The Fast Fourier Transform (FFT) was used to estimate the Discrete Fourier transform of each signal and the variance of the PSD was decremented through overlapping subsequent time windows of $50 \%$ (i.e., 0.5 s). For each task, we computed the power spectra in the $3 \mathrm{~s}$ window before the movement (i.e., when the subject imagines the upper limb movement to perform) within the canonical EEG frequency bandwidths: $\delta[0.5-4 \mathrm{~Hz}), \theta[4-8 \mathrm{~Hz}), \alpha[8-12 \mathrm{~Hz}), \beta$ $[12-30 \mathrm{~Hz}), \gamma[30-45 \mathrm{~Hz}]$.

\section{Classification}

In this preliminary study, in order to decrease the computational cost of the classification problem and reduce the risk of overfitting, we selected only the electrodes corresponding to the standard 10-20 system, plus a 20-th in the Oz position. Therefore, the input dataset was comprised of 1828 data examples ( 60 movements $\times 33$ subjects - corrupted discarded tasks), and 100 features (20 electrodes $\times 5$ bands).

To further reduce the number of features to be used for the classification, and to identify the most informative brain regions and EEG oscillations for the discrimination between the two classes (transitive vs. intransitive), we employed a standard nonlinear Support Vector Machine-Recursive Feature Elimination algorithm (SVM-RFE) [29]. This procedure implements an embedded feature selection procedure [29] together with a correlation bias reduction strategy. More specifically, at each iteration the less informative feature, according to the ranking criterion, was removed. On the remaining features an SVM with a radial basis function kernel was applied [29].

The classification algorithm was validated using a standard leave-one-movement-out cross-validation procedure (i.e., a variant of a leave-one-out cross-validation). This allowed the classifier to be trained while avoiding to be over-tuned due to multiple data samples of the same movement in the training set. More specifically, at each iteration of the cross-validation procedure, the input dataset was divided into a validation set, comprised of the three repetition of one movement performed 


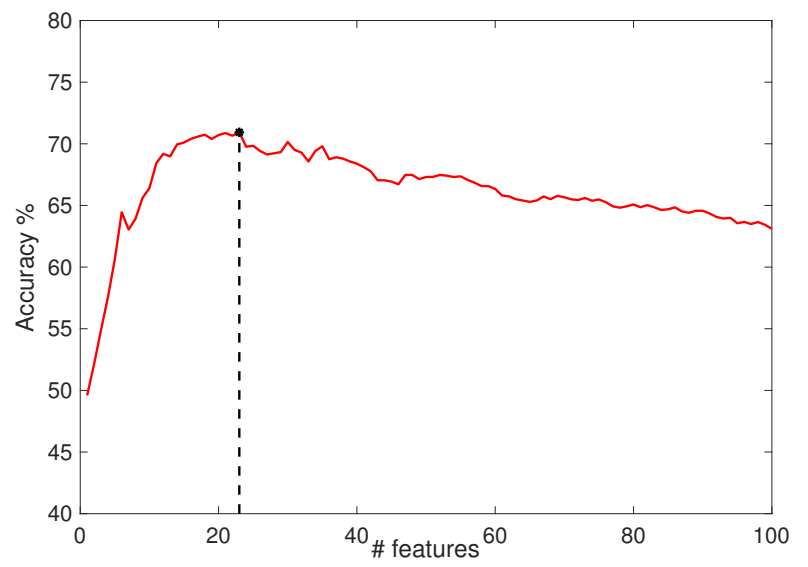

Fig. 3: Average accuracy over all cross validation. The best one has been obtained with 23 features and its value is $70.97 \%$

TABLE I: Confusion Matrix obtained with the most accurate (informative) subset of features

\begin{tabular}{|c|l|l|}
\hline & Classified Intransitive & Classified Transitive \\
\hline Intransitive & $\mathbf{7 7 . 2 7 7 6 \%}$ & $22.7224 \%$ \\
\cline { 2 - 3 } Transitive & $35.419 \%$ & $\mathbf{6 4 . 5 8 1 0 \%}$ \\
\hline
\end{tabular}

by a single subject, and a training set comprised of all the other samples.

In this study, the classification results are shown in the form of a confusion matrix calculated on the validation set. More specifically, a $2 \times 2$ table shows the percentage of true positive and true negative predictions along the diagonal. Therefore, a high average values on the diagonal means a more accurate classification.

\section{RESULTS}

Experimental results are presented as follows: first, we show the accuracy trend as a function of the number of features ranked through the SVM-RFE procedure. Then, a discussion of the brain areas and EEG oscillations associated with the best classification accuracy is reported.

\section{A. Classification accuracies}

Figure 3 shows the balanced average accuracies gathered at each iteration of the SVM-RFE algorithm, with increasing number of features. A maximum accuracy of $70.97 \%$ is reached with the 23 most informative features according to the ranking criterion. The associated confusion matrix is shown in Table I, with a sensitivity of $77.27 \%$ of and a specificity of $64.58 \%$.

\section{B. Feature-selection results}

The 23 most informative features (i.e., all selected electrode-band pairs that achieved the maximum averaged accuracy) are shown through topographic maps in Figure 4, separated by bands. The PSD of only fourteen out of twenty electrodes was in this set, i.e., the PSD computed in all the frequency bands of six electrodes was not selected among the first twenty-three positions of the feature ranking. Consequently, some of them were informative in more than a band. Among all the EEG bands, the most frequently selected EEG electrodes were located in the frontal and tempoparietal cortices. Remarkably, F4, in the pre-frontal right cortex, was selected over the $\theta, \alpha, \beta$ and $\gamma$ oscillations. Also, $\delta$ oscillations, which are usually considered as prominent in sleeping and non-conscience state, did not give a significant contribution for our motor imagery classification except for Pz. Conversely, oscillations in the $\theta$ and $\beta$ bands shared the selection of the right pre-motor and temporal regions, whereas $\alpha$ activity showed a discriminative power in the prominent frontal regions and in the occipital one. Indeed, transitive movements (where subjects interacted with objects) involved occipital regions, which are commonly related to visual tasks, more than the intransitive movements. The most discriminant oscillations between transitive and intransitive movements were in the $\gamma$ band. Results showed a symmetrical selection of the left and right prefrontal, frontal, and temporal regions. In addition, among the most informative features, there were also the $\gamma$-PSD of the $\mathrm{O} 1$ and P4 electrodes.

\section{DISCUSSION AND CONCLUSION}

In this preliminary study, we presented an automatic approach for transitive/intransitive movement recognition using information coming from EEG power spectra exclusively. Data were gathered from twenty electrodes, according to the standard 10-20 electrode placement system.

Thirty-three healthy volunteers were recruited in the study. They took part in an experiment where they have to imagine and act transitive and intransitive movements (according to [27]). We demonstrated that, with a rigorous feature selection stage combined with a SVM classifier, it is possible to recognise the two movement classes with an average accuracy of $70.97 \%$ (with a sensitivity of $77.27 \%$ and a specificity of $64.58 \%$ ). In addition, it is worthwhile noting that a satisfactory accuracy close to $70 \%$ was already achieved using a sub-set including only the first sixteen features.

The feature selection procedure revealed that the most relevant oscillations contributing to the maximal classification accuracy were in the $\gamma$ band. Note that, in many EEG studies dealing with movement analysis, the $\gamma$ band was poorly investigated because of the associated motion-artefacts. In our study, we minimised this issue by considering EEG data during motor imagery exclusively. Interestingly the activation of the contralateral hemisphere, which is commonly associated with motor control brain activity, revealed to be informative only in the $\gamma$ band. The other bands exhibited a more ipsilateral activation for the selected discriminant electrode subset. This result is in agreement with [21], which showed how oscillations in the $\alpha$ and $\beta$ bands over the ipsilateral cortex were involved in the hand representation, whereas the ones over the contralateral cortex were involved in the motor control. Note that in our study the motor control phase was not included, thus explaining the absence of these regions in our results. The more prominent differences in the 

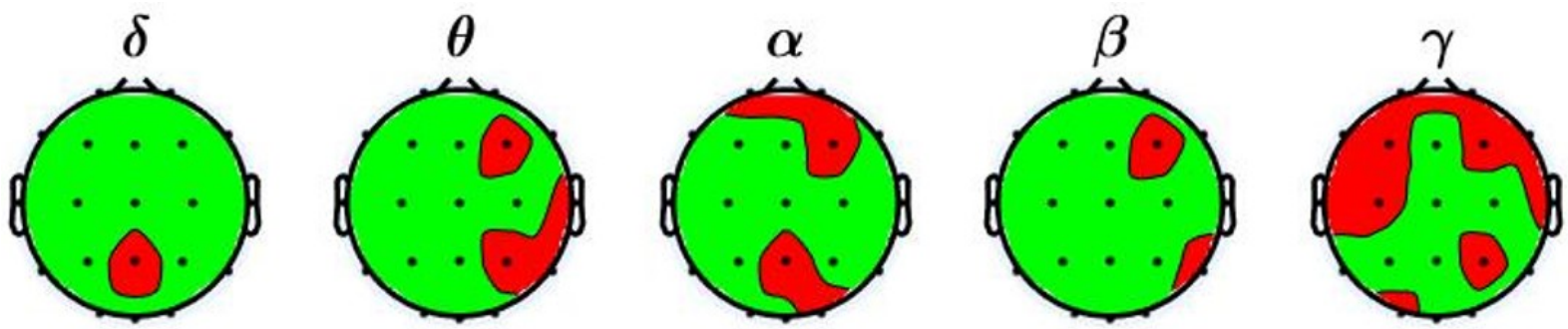

Fig. 4: Selected regions by the SVM-RFE algorithm for each EEG band.

two movement classes relied on a cognitive point of view, more than on the motor control itself. This can also explain the $\gamma$ band importance, with respect to the other bands, since $\gamma$-band is involved in high-level cognitive [30] and object representation tasks [31], [32].

The outcomes of the study can offer useful indications to be further pursued for the development of brain-machine interface, e.g. in terms of electrode placement and frequency bands to focus on for the correct imagination and execution of the movements. Future studies will be directed towards a comprehensive understanding of the cortical representation and control of upper limb voluntary movements, including also a tool mediated class. In addition, cortical oscillations during real movement will be investigated.

\section{REFERENCES}

[1] M. Santello et al., "Are movement disorders and sensorimotor injuries pathologic synergies? when normal multi-joint movement synergies become pathologic," Frontiers in human neuroscience, vol. 8, p. 1050, 2015.

[2] G. Averta et al., "Unvealing the principal modes of human upper limb movements through functional analysis," Frontiers in Robotics and AI, vol. 4, p. 37, 2017.

[3] C. Della Santina et al., "Postural hand synergies during environmental constraint exploitation," Frontiers in neurorobotics, vol. 11, p. 41, 2017.

[4] M. Gabiccini et al., "A data-driven kinematic model of the human hand with soft-tissue artifact compensation mechanism for grasp synergy analysis," in Intelligent Robots and Systems (IROS), 2013 IEEE/RSJ International Conference on, pp. 3738-3745, IEEE, 2013.

[5] J. Lenarcic et al., "Simple model of human arm reachable workspace," IEEE transactions on systems, man, and cybernetics, vol. 24, no. 8, pp. 1239-1246, 1994.

[6] G. Rau et al., "Movement biomechanics goes upwards: from the leg to the arm," Journal of biomechanics, vol. 33, no. 10, pp. 1207-1216, 2000.

[7] H. S. Lo et al., "Exoskeleton robots for upper-limb rehabilitation: State of the art and future prospects," Medical Engineering and Physics, vol. 34, no. 3, pp. 261-268, 2012.

[8] A. Bartolo et al., "Cognitive approach to the assessment of limb apraxia," The Clinical Neuropsychologist, vol. 22, no. 1, pp. 27-45, 2008.

[9] L. J. Buxbaum et al., "Critical brain regions for tool-related and imitative actions: a componential analysis," Brain, vol. 137, no. 7, pp. 1971-1985, 2014.

[10] M. Lotze et al., "fmri evaluation of somatotopic representation in human primary motor cortex," Neuroimage, vol. 11, no. 5, pp. 473481, 2000.

[11] A. R. Luft et al., "Comparing brain activation associated with isolated upper and lower limb movement across corresponding joints," Human brain mapping, vol. 17, no. 2, pp. 131-140, 2002.

[12] F. Binkofski et al., "The role of ventral premotor cortex in action execution and action understanding," Journal of Physiology-Paris, vol. 99, no. 4-6, pp. 396-405, 2006.
[13] I. Miyai et al., "Cortical mapping of gait in humans: a nearinfrared spectroscopic topography study," Neuroimage, vol. 14, no. 5, pp. 1186-1192, 2001.

[14] R. Hari, "Action-perception connection and the cortical mu rhythm," Progress in brain research, vol. 159, pp. 253-260, 2006.

[15] R. Salmelin and R. Hari, "Spatiotemporal characteristics of sensorimotor neuromagnetic rhythms related to thumb movement," Neuroscience, vol. 60, no. 2, pp. 537-550, 1994.

[16] P. Avanzini et al., "The dynamics of sensorimotor cortical oscillations during the observation of hand movements: an eeg study," PLoS One, vol. 7, no. 5, p. e37534, 2012.

[17] G. Pfurtscheller et al., "Evaluation of event-related desynchronization (erd) preceding and following voluntary self-paced movement," Clinical Neurophysiology, vol. 46, no. 2, pp. 138-146, 1979.

[18] C. Neuper et al., "Erd/ers patterns reflecting sensorimotor activation and deactivation," Progress in brain research, vol. 159, pp. 211-222, 2006.

[19] G. Pfurtscheller et al., "Motor imagery activates primary sensorimotor area in humans," Neuroscience letters, vol. 239, no. 2-3, pp. 65-68, 1997.

[20] G. Rizzolatti et al., "The mirror-neuron system," Аnnи. Rev. Neurosci., vol. 27, pp. 169-192, 2004.

[21] T. Ball et al., "The role of higher-order motor areas in voluntary movement as revealed by high-resolution eeg and fmri," Neuroimage, vol. 10 , no. 6, pp. 682-694, 1999.

[22] S. Caspers et al., "Ale meta-analysis of action observation and imitation in the human brain," Neuroimage, vol. 50, no. 3, pp. 1148-1167, 2010.

[23] D. Arnstein et al., " $\mu$-suppression during action observation and execution correlates with bold in dorsal premotor, inferior parietal, and si cortices," Journal of Neuroscience, vol. 31, no. 40, pp. 1424314249, 2011.

[24] P. Molenberghs et al., "Brain regions with mirror properties: a metaanalysis of 125 human fmri studies," Neuroscience \& Biobehavioral Reviews, vol. 36, no. 1, pp. 341-349, 2012.

[25] B. Petreska et al., "Apraxia: a review," Progress in brain research, vol. 164, pp. 61-83, 2007.

[26] L. Canzano et al., "The representation of objects in apraxia: From action execution to error awareness," Frontiers in human neuroscience, vol. 10, 2016.

[27] G. Handjaras et al., "A topographical organization for action representation in the human brain," Human brain mapping, vol. 36, no. 10, pp. 3832-3844, 2015.

[28] A. Delorme et al., "Eeglab: an open source toolbox for analysis of single-trial eeg dynamics including independent component analysis," Journal of neuroscience methods, vol. 134, no. 1, pp. 9-21, 2004.

[29] K. Yan et al., "Feature selection and analysis on correlated gas sensor data with recursive feature elimination," Sensors and Actuators B: Chemical, vol. 212, pp. 353-363, 2015.

[30] C. S. Herrmann et al., "Cognitive functions of gamma-band activity: memory match and utilization," Trends in cognitive sciences, vol. 8, no. 8, pp. 347-355, 2004.

[31] C. Tallon-Baudry et al., "Oscillatory gamma activity in humans and its role in object representation," Trends in cognitive sciences, vol. 3 , no. 4, pp. 151-162, 1999.

[32] G. Valenza, A. Greco, M. Bianchi, M. Nardelli, S. Rossi, and S. E. Pasquale, "EEG Oscillations during Caress-like Affective Haptic Elicitation," Psychophysiology, 2018. 